\title{
DEVELOPMENT AND TESTING TOOL FOR PAPER HANDLING AND/OR PRINTER DEVICES
}

\author{
N. V. Castillo', D.Torres' \& H. Gutiérrez² \\ ${ }^{1}$ CINVESTAV del IPN GDL \\ ${ }^{2}$ Hewlett Packard de Mexico \\ Received: February $13^{\text {th }} 2001$ and accepted March $5^{\text {the }} 2002$
}

\section{ABSTRACT}

A useful development and testing tool for Hewlett Packard paper handling and/or printerdevicesispresented. The system provides a microprocessor based general architecture integrated by two PCB cards and a software application, used to improve new product design and testing. Paper handling devices operation is based on DC motors, stepper motors and sensors components, so a graphical user interface was developed in order to configure, drive, and test them. The implemented tool allows a time reduction greaterthan $50 \%$ for the development and test of prototypes.

\section{RESUMEN}

Se presenta una herramienta para la prueba de dispositivos Hewlett Packard de manejo de papel y/o impresión. El sistema tiene una arquitectura de microprocesamiento integrada por dos tarjetas PCB y un programa para mejorar el diseño y la prueba de nuevos productos. La operación de los dispositivos de manejo de papel está basado en motores DC, motores de pasos y sensores, por lo que se desarrolla una interfaz de usuario gráfico para configurarlos, manejarlos y probarlos. Esta herramienta permite una reducción de más del $50 \%$ del tiempo de desarrollo y pruebas de prototipos.

KEYWORDS: Tool development, paper handling and printer devices, hard-software codesign, testing

\section{INTRODUCTION}

During the last years, some companies have developed high efficient printers, e.g. Hew lett Packard, in order to satisfy large printer offices demands. By means of paper handling devices -PHDs-, new est printers are able to separate, distribute, and staple documents. In this way, most PHDs connected at printer's paper interfaces -input and output-, are designed to move paper sheets $w$ ith a high precision.

In general, the development process of electronic devices consists of several levels: conception, analysis and design, implementation, and testing. For the development of similar products, the same process is alw ays follow ed. Due to delivery of electronic devices is critic in time, reusable control architecture is desired in order to decrease time intervals betw een prototypes and final products. This is possible for a device family, w here products are based on similar functionality and operation procedures.

All PHDs operation is based on electromechanical components, e.g. motors and sensors. According to this, generic control architecture should be created to simplify their developing procedures. In order to provide such functionality, the platform must be able to satisfy the follow ing requirements:

1. To generate any system paper sheet movement, providing every signal required moving and controlling DC and stepper motors.

2. To determine, at any time, paper sheets position, through electronic sensors operation. 
3. To store and to execute programmable code (firmw are), in a microprocessor based system.

4. To provide internal printer communication, using CAN 2.0B standard protocol.

5. To support PC communication, through RS232_C protocol.

6. To allow system-user interaction, based on a graphical softw are program running over a PC.

Main objectives of the present w ork are:

1. To design a reusable architecture for control and operation of printer paper handling devices.

2. To implement the tool for development and testing of the printer devices.

\section{HEWLETT PACKARD PAPER HANDLING DEVICES -PHDS-}

PHDs are attached to printers and they take the printed paper from the printer and, depending on the type of device, either puts it into a high capacity output tray, or into a collation mechanism to separate, distribute or staple documents. Most printers provide tw o output trays; one "face up" and one "face dow $\mathrm{n}$ " to send their printed jobs. Fig. 1 show s specific HP printers' output paper trays [1].

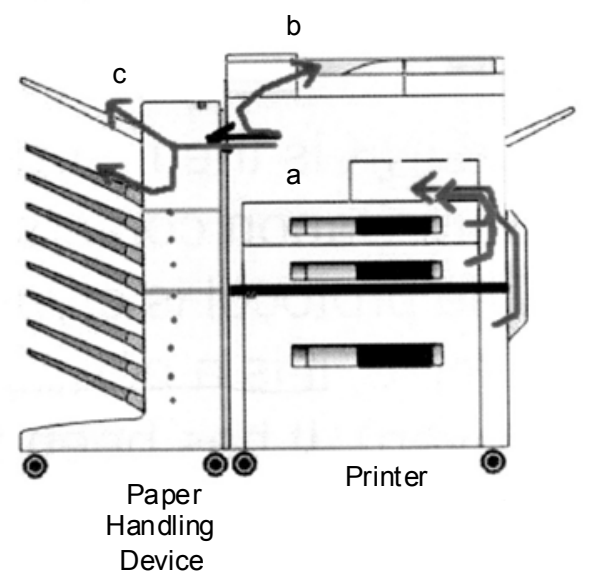

Figure1. Hewlett Packard printer output paper trays

In addition to normally output paper containers, HP printers have the capability to attach PHDs to receive the printed paper. They have several output additional trays, selectable by command, allow ing the separation of different print jobs. Paper handling devices stack sheets and provide automatic job offset capabilities for easy handling and job separation. It also allow s in line stapling, enabling the creation of a wide range of documents ready for distribution in just one step [1].

\subsection{Type of papers}

For HP printers and PHDs the best paper has the follow ing characteristics [2]:

Cleanly and accurately cut.

Paperw eight of around 80-90 gsm (grams per square meter).

Dry and free of dust.

Other type of papers contaminates the printer mechanism, and may skew or crease in the paper path. 
Frequently is necessary to print documents over different paper types. HP printers and paper handling devices allows the use of plasticized paper, cards, transparencies, and other types of special printing materials, $w$ ith different paper densities.

\subsection{Paper Movement Analysis}

Different paper movements, on paper handling devices, can be described by the set $T=\{T 1, T 2 \ldots T n\}$, where $\mathrm{Ti}$ is a trajectory determining sheet of paper path. For every $\mathrm{Ti}$, each sheet is moved fromprinter to the selected output paper container. In order to improve the transportation through devices of sheets of paper, movement profiles $\mathrm{Mi}$ are introduced and associated to every Ti paper trajectory. Besides, each movement profile $\mathrm{Mi}$ is a set $\mathrm{Mi}=\{\mathrm{m} 1, \mathrm{~m} 2 \ldots \mathrm{mk}\}$, where $\mathrm{mk}$ are independent elementary movements. These movements are related $w$ ith DC motors, stepper motors, and sensors. These are components denoted by en. Then, it is possible to consider a function, $\mathrm{fk}$, able to generate any $\mathrm{mk}$ required for a printer device; and consequently, $\mathrm{mk}=\mathrm{fk}(\mathrm{e} 1, \mathrm{e} 2 \ldots \mathrm{en})$. Paper movements require, inside of printer devices, of:

High precision and minimum load movements.

Realization device critical operations: such as supervision and regulation of paper movement.

Fig. 2 show s the principles of DC and stepper motors. Generally, motors are used through gears and bands system, which permits to obtain desired speed of the sheet of paper. The systemalows reaching motor operation close to some optimal parameters that produce the rollers spinning and therefore paper movement. Rollers provide a proportional force originated by their ow $n$ attraction force and a fixed frictional coefficient. Under optimal conditions totally arrow angular movement is transferred to the paper, producing a linear movement. By this way, paper speed and acceleration are directly controlled by the motor operation.

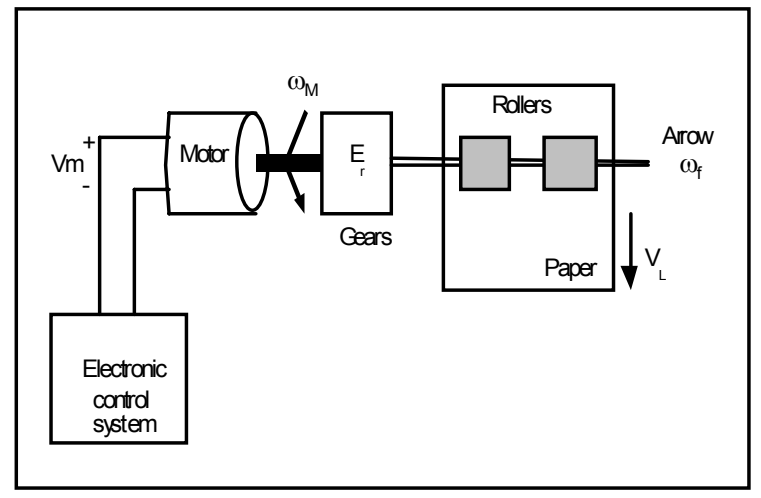

Figure. 2 DC and stepper motors scheme

\subsubsection{DC motors}

According to Fig. 2, the lineal speed, $v L$, is determined by $v L=P w f$ equation, where $P=2 p R r$, represents the attraction force betw een the rollers of radio $\mathrm{Rr}$ over every sheet of paper. Besides, the variable $\mathrm{w} f$ represents arrow final angular speed, which is directly proportional to gears relationship $E$, and its motor angular speed $w \mathrm{M}(\mathrm{wf}=\mathrm{Erw} \mathrm{M})$ [3]. The $\mathrm{w} \mathrm{M}$ value is directly proportional to voltage applied to the motor, VM, and to manufacturer factor KM, i.e., w $\mathrm{M}=\mathrm{VMKM}$. So the final lineal speed could be described by the next equation:

$$
v_{L}=2 \pi R_{r} E_{r} V_{M} K_{M}
$$

The eq. 1 show s the relationship betw een the voltage applied to the motor, VM, and the system lineal speed, vL. Final paper lineal movement, $M L$, is a function of $v L$, and time. The variable time is introduced 
using the step function $\mathrm{m}$ (t-to) to describe the paper movement over time intervals. Finally, the paper movement is given by the relationship:

$$
M_{L}=v_{L} \mu\left(t-t_{o}\right)
$$

Consequently, the voltage applied to the motor is directly related to the final paper movement lineal speed. The polarity of the voltage regulates the motor movement direction.

\subsubsection{Stepper motors}

Stepper speed and movement analysis is presented in reference [4]. For this case, substituting ineq. (1) the voltage applied to the motor, VM, by a frequency source $\mathrm{f}$, and the manufacturer factor, $\mathrm{KM}$, by the equation $\mathrm{KM}=2 \mathrm{p} / \mathrm{n}$, w here $\mathrm{n}$ represents the steps number required to reach a full motor revolution. $\mathrm{t}$ is obtained that paper lineal speed is given by:

$$
M_{L}=v_{L} \mu\left(t-t_{o}\right)
$$

In addition, stepper motors have capabilities to maintain a constant force over rollers, allow ing sheet of paper required fixed position, in order to avoid movement failures. Stepper motor movement direction is given by the introduction of a sequence of the electrical pulses.

\subsection{Movement and speed profiles}

Fig. 3 show $s$ the acceleration, speed and movement profiles of paper sheet. The acceleration profles during pre-movement stages of paper (ã1, ã3 and ã5) proportionate to DC and stepper motors, the required time to find their optimal operation parameters. These parameters determine the lineal speed levels required moving paper through devices and their values are very important during the product development process.

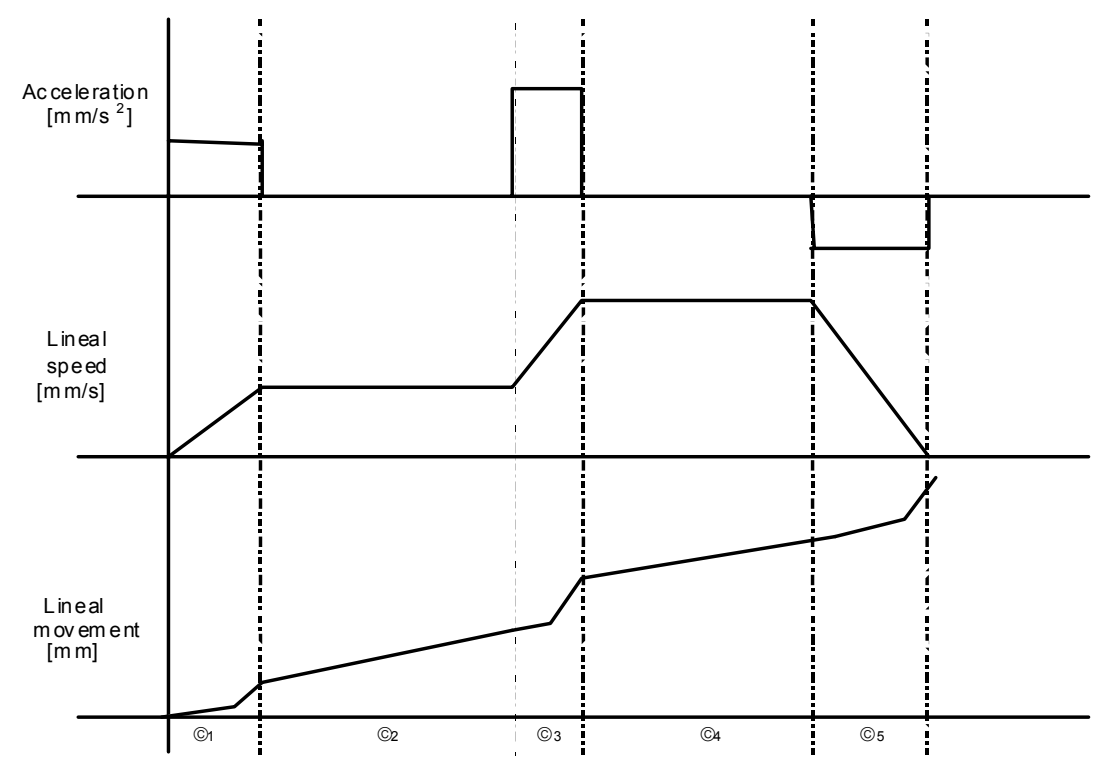

Figure 3. Acceleration, speed and movement profiles of paper sheet.

The acceleration interval duration ã1, ã3, ã5, can be neglected in comparison w ith the sheets of paper transport timings given by the periods ã2, ã4. This means that all this acceleration intervals prepare the electromechanical system to carry out the sheets of paper through the device. 


\section{ARCHITECTURE}

PHDs, as most electronic products, are implemented by using programmable components. Microprocessors and peripheral components, such as controllers and special application modules [5], define their architecture. These application modules are w ell-defined subsystems to control motors and handle sensors. In this way, a generic architecture can be created and it can be used in other implementations. Therefore, engineers $w$ ill use the tool in order to develop current devices in shorter time intervals than before.

The selected architecture for the tool is show $n$ in Fig. 4. It consists of the follow ing parts: hardw are, control firmw are, user application softw are, and communication interfaces.

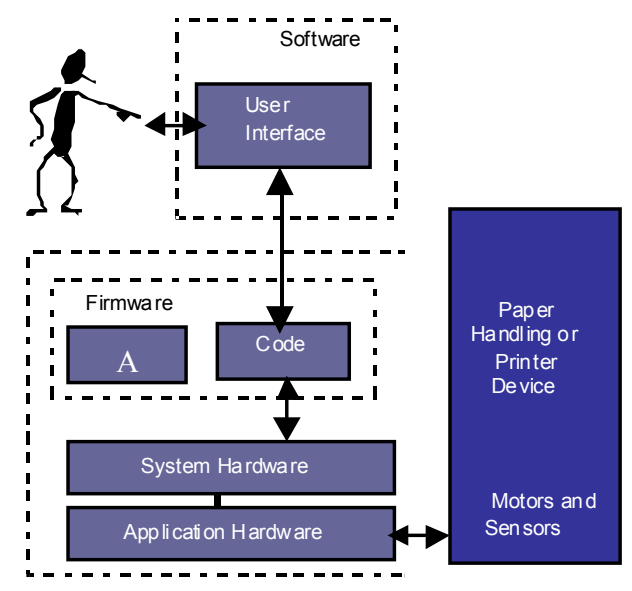

Figure 4. Paper handling and printer device development tool architecture.

\subsection{Hardware}

It has every required physical device to move motors, read sensors and execute special tasks. A microprocessor, memories, controllers, motor drivers, communication circuits and glue logic form this block. It is divided in tw o PCB cards: the first one is called system hardw are card, and the second one application hardw are card.

System Hardw are Card. It is the control system core for the development and testing tool. Besides, tis based on a programmable microprocessor, w hich is able to realize a group of general-purpose tasks, which are required to implements paper handling devices functionality (execute programmable code, external communication interfacing).

Application Hardw are Card. Its principal function is to support every physical device necessary tomove motors and read sensors. Through a connector, it is attached to the system hardw are card.

\subsection{Control Firmware}

The firmw are constitutes the softw are interface betw een hardw are and application softw are. It is a programmable instruction set embedded into the microprocessor. This set of instructions handle and control all the input and output signals related to microprocessor, that are required to execute thesystem desired operations [6].

Control firmw are is divided in two basic components. The first one is a set $A=\{A 0, A 1, \ldots A n\}$ of dedicated algorithms Ak; and the second one is the access code CA. Algorithms Ak are used tomove DC and stepper motors, originating elementary movements $\mathrm{mk}$, which help to build every movement profile Mi and, consequently the paper trajectory $\mathrm{Ti}$. The access code CA has tw o differentfunctions: to 
select an algorithm Ak, and to communicate w ith the application softw are running into a PC over the interface RS_232_C (at speeds of up to 38400 bps). All the information used and generated by this module is interpreted and received by the third functional block: the user application softw are.

\subsection{User Application Software}

User application softw are runs over PC Window s's 95/98/NT operating systems. Besides, the program implements serial port communication $w$ ith the control firmw are, it provides a user graphical interface. $t$ was developed using C++ Builder 3.0 tool and object oriented programming. All the generated information is coded and transferred to the control firmw are; then hardw are components execute the desired operations. Softw are must provide to the engineers $w$ ith a set of required commands to configure and test devices, e.g. DC-, stepper motors, and sensor components, according to the application.

\subsection{Communication Interface}

All PHDs must realizes tasks such as signal detecting, component configuration, motors activation and deactivation, and activities related to device paper movement. Although, operation of most devices is determined by external variables coming up from printers or other printer controllers; a PHD need to have a communication protocol in order to know all their w orking conditions. According to this, HP engineers adopted CAN version 2.0B as a standard protocol to define their PHDs and printer interfaces.

\subsubsection{CAN 2.OB Protocol}

The Controller Area Netw ork (CAN) is a serial communications protocol of high integrity, which efficiently supports distributed real-time control w ith a very high level of security. Its domain of application goes from high-speed netw orks to low cost multiplex w iring, -see Fig. 5. It operates at data rates of up to 1 $\mathrm{Mb} / \mathrm{s}$, providing an excellent error detection and reliability. CAN is documented in ISO 11898 (for highspeed applications) and ISO 11519-2 (for low er-speed applications) [7]. It has the following characteristics:

CAN is a lineal multi-master bus $w$ ith one logic bus line and equal nodes.

Their bus nodes do not have a specific address.

Instead of it, the identifiers of the transmitted messages, indicating the message content and the priority of the message carry out the address information. The higher priority message is guaranteed to gain bus access as if it were the only message being transmitted. Low er priority messages are automatically retransmitted in the next bus cycle or in a subsequent bus cycle if there are still other higher priority messages $w$ aiting to be sent [8].

The number of nodes is not limited by the protocol and can be changed dynamically without disturbing the communication betw een nodes.

To support multicast and broadcast addressing.

To provide sophisticated error-detection and error handling mechanism such as CRCcheck, and high immunity against electromagnetic interference. Erroneous messages are automatically retransmitted, and temporary errors are recovered.

Non-return-to-Zero code. For synchronization purposes, bit stuffing is used.

There is a high data transfer rate up to $1 \mathrm{Mb} / \mathrm{s}$, at buses length of 40 meters using tw istedwire pair. Messages length has a maximum of 8 data bytes. 
The bus access is handled via the advanced serial communications protocol Carrier Sense Multiple Access/Collision Detecting w ith Non-Destructive Arbitration.

CAN is implemented in some commercial chips.

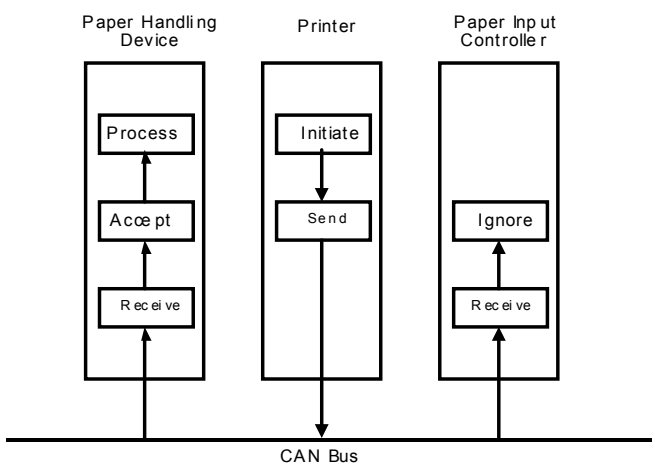

Figure 5. CAN bus: Messages transmission on the printer and paper handling devices

A development and testing tool for PHDs must integrate every necessary component to fulfill CAN2.0B specifications. Through CAN interface each device $w$ ill be communicated $w$ ith current printer. At the same time, designed tool must support RS_232_C protocol in order to communicate the PHD controller $w$ ith the application softw are running over any PC.

\section{HARDWARE}

As w e already mentioned, tw o PCB cards represent the hardw are block: the systemhardware card and the application hardw are card.

System Hardw are Card. Microprocessor and peripheral components selection defines paper handling hardw are design. This card interfaces w ith other devices (see Fig. 6). Main components are described below :

16-bit H8S/2357 Hitachi high efficient microprocessor [9].

2 Mbit of Flash memory organized as $128 \mathrm{~K}$ x 16 bits, 512 Kbits of Static RAM organized as $32 \mathrm{~K}$ x 16 bits, and 512 bytes of NVRAM (serial EFPROM).

CAN 2.0B communication protocol controller.

Debug port through RS232 driver.

System hardw are card implementation gives development tool capabilities to:

Execution of general-purpose tasks.

CAN 2.0B access, enabling printer and paper handling devices communication.

PC serial port interface. 


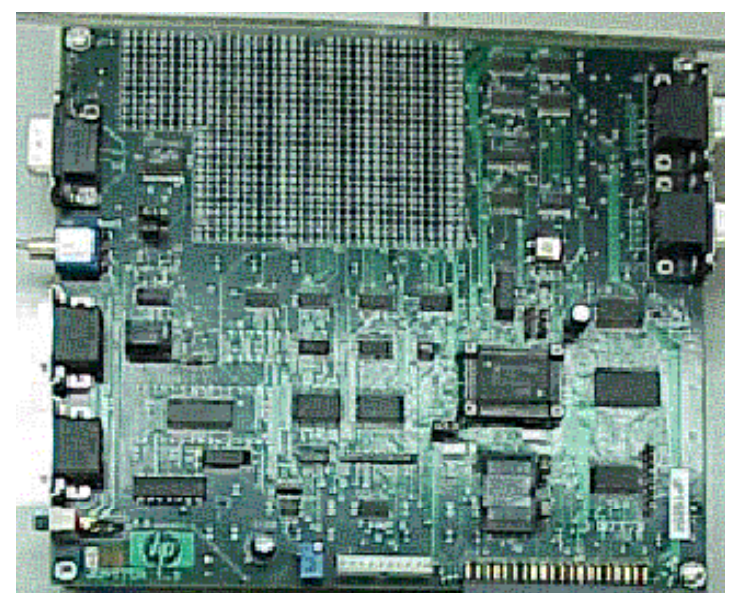

Figure 6. Development Tool: System hardware PCB card.

Application Hardw are Card. It is attached to the system hardw are card. It w as designed to representa kind of universal board to control steppers and DC motors. It also has the capability to read sensors and to generate an interrupt $w$ hen any of them changes from active to inactive or vice versa (see Fig. 7). A list of all features included in this card is:

40-pin system hardw are card connector.

Necessary logic to read up to 16 sensors.

Circuit EPMT064S PLD (programmable logic device) to implement the sensor interrupts [10].

Drivers to handle and control up to 14 DC motors and 31 stepper motors.

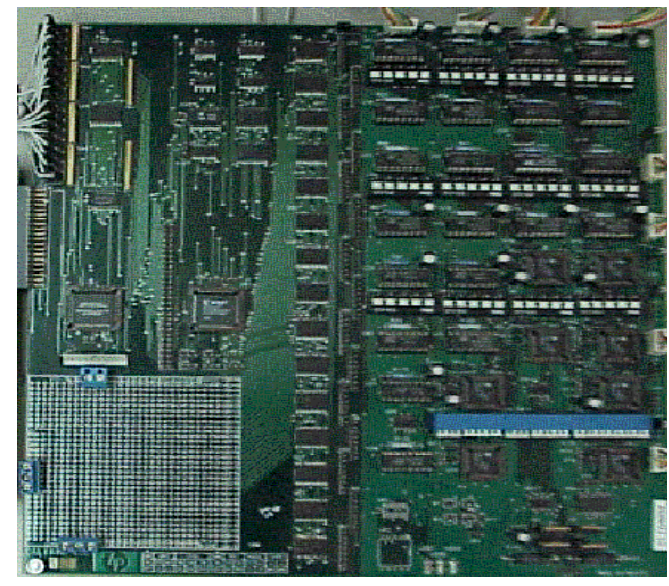

Figure 7. Development Tool: Application hardware PCB card.

\section{SOFTWARE}

User application softw are provides an interactive interface to developer engineers to configureand test their devices of any electromechanical system. By the GUI interface, users are able to drive DCmotors, stepper motors and sensors according to the requirements, in order to find the optimal operation parameters of many devices. 
According to this functionality the softw are, based on object oriented programming, was developed using $\mathrm{C}++$ Builder 3.0 tool [11] and it is integrated by the elements show $\mathrm{n}$ in Fig. 8.

Control. Principal softw are component implemented to control and supervise the program functionality:

a) To program environment setup, creating all the functional softw are objects required to reflect every development tool hardw are component.

b) To implement the user graphical interface.

c) To create, update and control all system active elements (DC-, stepper motors, and sensors), according to firmw are and user interaction.

d) To capture and code user data to be transmitted and executed by dedicated controlfrmware. At the same time, coming up firmw are data decoding and interpretation. Serial port driver implementation to receive and send data through RS_232_C protocol.

Functional Objects. Components are classified according to following statements. Any hardw are representative object forms first group such motor driver chips and sensors electronic components. The second group describes all the software motors and sensors logical representation, which are dynamically activated and deactivated during program execution.

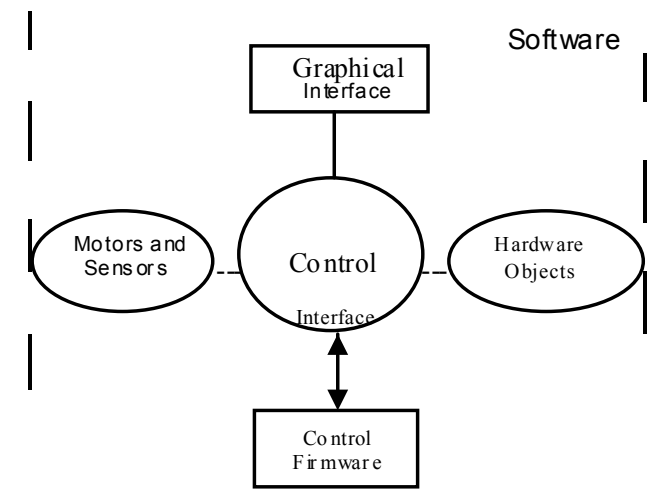

Figure 8. User Application Software Architecture

Graphic Interface. An intuitive and ease of use interface, see Fig. 9, allow s to the user handle up to 30 DC motors, 14 stepper motors and 16 sensors [12]. Users can handle independently each motor, adjust the level current parameters, fix different speed levels to determine stepper motors operation, userdefined ramps up (acceleration) and ramps dow $n$ (deceleration) in order to accomplish different paper movements, and read sensor logical level.

\subsection{Softw are Characteristics:}

Softw are was developed using object-oriented techniques and the C++ Builder 3.0 tool. It runs over Window s 95/98/NT operating system. Six ow n classes w ere implemented, 22 recipient classes used, 2050 code lines w ritten, 157000 compiled lines, and the executable program has a volume of $475 K b y t e s$. 


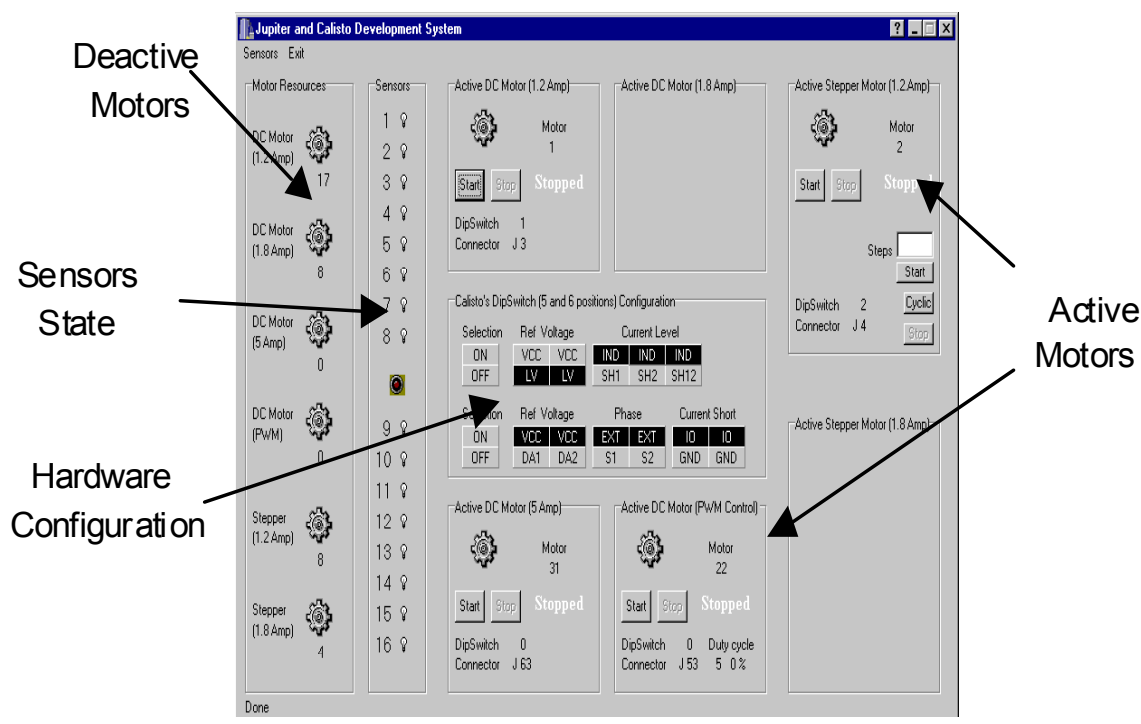

Figure 9 User Application Software Interface

\section{RESULTS AND ANALYSIS}

A paper handling device consists of different parts: mechanical, electronic and firmw are. During the normal development process, the mechanical part w as implemented necessarily before the other ones. The debugging process $\mathrm{w}$ as very difficult. Besides, the process to find good movement profles was very time consuming, due to the load and unload of different algorithms using traditional methods. For these reasons, the development time was approximately $16 \mathrm{w}$ eeks.

In the present $w$ ork the development process $w$ as studied to reduce its duration. Therefore, relationships betw een trajectories, movement profiles, elementary movements, components and functions were analyzed.

Movements of piece of papers through printers and PHDs can be described by trajectories Ti. There is a movement profile Mi associated with each trajectory Ti .

An ordered set of independent elementary movements $\mathrm{mk}$ builds a movement profile $\mathrm{Mi}$, and every $m k$ required for a printer device is expressed as a function $m k=f k(e 1, e 2 \ldots e n)$, where eidenotes a component.

Tw o basic components of the firmw are were implemented. The first one is a set $A=\{A 0, A 1$, ...An\} of dedicated algorithms Ak and, the second one is the access code CA. Algorithms Akare used to build every movement profile $\mathrm{Mi}$ and, consequently the paper trajectory $\mathrm{Ti}$.

Some metrics such as: number of printed pages per minute and, number of jammed sheets are very important to evaluate a trajectory $\mathrm{Ti}$ and its movement profile Mi.

The new implemented platform or tool contributes to the automation of the development process of PHDs and printer devices allow ing to the engineer:

To achieve a flexible composition of different movement profile Mi for research purposes.

To find good movement profiles for printer devices and PHDs, saving time. 

before.

To w ork on firmw are in parallel w ith the mechanical part allow ing a greater independence than

For these reasons testing time to put in order a PHD device was decreased from 16 w eeks to only 4 w eeks. Accumulated experience by engineers using this tool continues decreasing this time interval.

\section{CONCLUSIONS}

A development and testing tool for paper handling and/or printer devices $\mathrm{w}$ as implemented. This platform simplifies and reduces efficiently the design and implementation of final Hew lett Packard products.

For the hardware were designed and built tw o PCB's denominated system hardw are board and application hardw are board. An EPM7064S PLD (Altera) w as included and programmed adding special system functionality to the hardw are. The microprocessor firmw are $w$ as $w$ ritten in Clanguage. lcontains the set A of dedicated algorithms and the access code CA. Algorithms Ak are used to move DC and stepper motors, originating elementary movements $\mathrm{mk}$, which help to build every movement profile $\mathrm{Mi}$ and, consequently the paper trajectory Ti. The access code CA has tw o different functions: to selectan algorithm Ak, and to communicate $w$ ith the application softw are.

The application softw are w as implemented using object-oriented techniques and it has a GUI Interface. By this dedicated graphical interface, users are able to configure, operate and control any motor and sensor connected to the platform, in order to find optimal operation parameters required to movesheets of paper through PHDs.

Development tool capabilities are related to implement general purpose tasks, access to CAN 2.0B standard protocol, maintain successful communication w ith a PC, recognize sensors logical levels, and handle DC motors and stepper motors.

The use of this tool for the development and testing of paper handling and/or printer devices has demonstrated a high usefulness and quality to release in shorter period of time high quality products. The first tests reduced the development and testing time from 16 to $4 \mathrm{w}$ eeks.

Besides, it represents a new platform to research and development optimal movement profles for printer devices and development tools for both R\&D (Research and Development) laboratory and service in the field for the support engineers at Hew lett Packard.

\section{ACKNOWLEDGEMENTS}

The authors wish to express their gratitude to Hew lett Packard for the financial support given to this w ork, and in particular to Mr. H. Rodríguez.

\section{REFERENCES}

[1] Hew lett Packard, Hardw are Drivers Documentation for Paper Handling Devices (Guadalajara), 158, (2000).

[2] Burrow s S., 1994 A Laser Printer Book, Ed. Black Lightning Inc, (New York), 5-25.

[3] Reliance Motion Control Inc, DC Motors, Speed Controls and Servo Systems Handbook, Ed. Reliance Inc, (Minnesota), 2-114, (1996).

[4] Ericsson Components, Stepper Motor Control Handbook, Ed. Ericsson, (Sw eden), 11-82, 1995.

[5] Merrill C., 1984 Microprocessor Hardw are/Softw are Interfacing, Ed. B. Brey. 
[6] Hew lett Packard, Paper Handling Firmw are Development Standard, (Guadalajara), 1-32, (2000).

[7] Bosch R., 1991 CAN Specification version 2.0, (Stuttgart), 2-68, ().

[8] Philips Semiconductors, Interfacing a Stand Alone CAN controller, (Amsterdam), 1-66, (2000).

[9] Hitachi Semiconductors, H8S/2357 Series, Hardw are Manual, (Tokyo), 1-1084, (1998).

[10] Altera, Max Plus II, Programmable Logic Development System, (San Jose), 12-300, (1995).

[11] Liberty J., 1998 Beginning Object-Oriented Analysis and Design: With C++, Ed. Wrox Press Inc, 35-68.

[12] Schildt H., 1997Borland C++, Ed. McGraw Hill, 55-255. 
Authors Biography

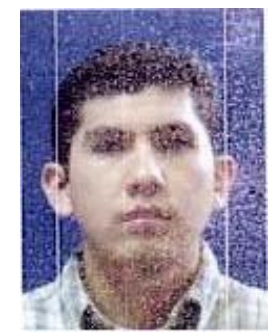

Vladimir Castillo

Received the B.E. degree in Electronics from Instituto Tecnologico de Sonora (ITSON) in 1998 and the M.S. in Telecommunication from Research Center and Advanced Studies of IPN, Mexico, in 2000. Since 2000 he is a member of R\&D Department of Hew lett Packard, Guadalajara, Mexico, w here he w orks w ith hardw are and firmw are for Printers.

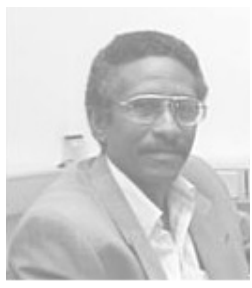

Deni Torres Román

Received a Ph.D. degree in telecommunication from Technical University Dresden, Germany in 1986. He w as professor at the University of Oriente, Cuba. Co-author of a book about Data Transmission. He received the Telecommunication Research Prize in 1993 from AHCIET Association and w as recipient of the 1995 best Paper Aw ard from AHCIET Review, Spain. Since 1996 he is associate professor at Research and Advanced Studies Center of IPN (Cinvestav- Mexico). He is a member of the IEEE.

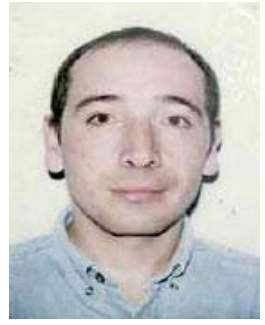

Hernán Gutiérrez

Received the B.E degree in Electronics in 1990 and the M.S. in Industrial Electronic from Instituto Tecnologico de Estudios Superiores de Occidente (ITESO), Mexico, in 1996. From 1990 to 1996 he w orked at different companies as IIE and GPI Mexican. Since 1996 he is a member of R\&D Department of Hew lett Packard, Guadalajara, Mexico, w here he w orks w ith hardw are and firmw are for Printers. 